\title{
On $k$-circulant matrices with the generalized third-order Pell numbers
}

\author{
Yüksel Soykan \\ Department of Mathematics, Art and Science Faculty, \\ Zonguldak Bülent Ecevit University \\ 67100, Zonguldak, Turkey \\ e-mail: yuksel_soykan@hotmail.com
}

Received: 18 January $2021 \quad$ Revised: 18 October $2021 \quad$ Accepted: 4 November 2021

Abstract: In this paper, we obtain explicit forms of the sum of entries, the maximum column sum matrix norm, the maximum row sum matrix norm, Euclidean norm, eigenvalues and determinant of $k$-circulant matrix with the generalized third-order Pell numbers. We also study the spectral norm of this $k$-circulant matrix. Furthermore, some numerical results for demonstrating the validity of the hypotheses of our results are given.

Keywords: Third-order Pell numbers, Circulant matrix, $k$-circulant matrix, Tribonacci numbers, Norm, Spectral norm, Determinant.

2020 Mathematics Subject Classification: 11B39, 11B83, 15A18, 15A60, 15B36, 11C20.

\section{Introduction}

In this section, we recall definitions and some properties of the generalized Tribonacci sequence and generalized third order Pell sequence. The generalized Tribonacci sequence

$$
\left\{W_{n}\left(W_{0}, W_{1}, W_{2} ; r, s, t\right)\right\}_{n \geq 0}
$$

(or shortly $\left\{W_{n}\right\}_{n \geq 0}$ ) is defined as follows:

$$
W_{n}=r W_{n-1}+s W_{n-2}+t W_{n-3}, \quad W_{0}=a, W_{1}=b, W_{2}=c, n \geq 3
$$

where $a, b, c$ are arbitrary complex (or real) numbers (not all being zero) and $r, s, t$ are real numbers (not all being zero). For more information on this sequence, see for example [21]. 
The sequence $\left\{W_{n}\right\}_{n \geq 0}$ can be extended to negative subscripts by defining

$$
W_{-n}=-\frac{s}{t} W_{-(n-1)}-\frac{r}{t} W_{-(n-2)}+\frac{1}{t} W_{-(n-3)}
$$

for $n=1,2,3, \ldots$ when $t \neq 0$. Therefore, recurrence (1) holds for all integer $n$.

Now we consider the case $r=2, s=t=1$ and in this case we write $V_{n}=W_{n}$. A generalized third order Pell sequence $\left\{V_{n}\right\}_{n \geq 0}=\left\{V_{n}\left(V_{0}, V_{1}, V_{2}\right)\right\}_{n \geq 0}$ is defined by the third-order recurrence relations

$$
V_{n}=2 V_{n-1}+V_{n-2}+V_{n-3}
$$

with the initial values $V_{0}=c_{0}, V_{1}=c_{1}, V_{2}=c_{2}$ where $c_{0}, c_{1}$ and $c_{2}$ are arbitrary real numbers (not all being zero).

The sequence $\left\{V_{n}\right\}_{n \geq 0}$ can be extended to negative subscripts by defining

$$
V_{-n}=-V_{-(n-1)}-2 V_{-(n-2)}+V_{-(n-3)}
$$

for $n=1,2,3, \ldots$. Therefore, recurrence (2) holds for all integer $n$.

Next, we define two special case of the sequence $\left\{V_{n}\right\}$. Third-order Pell sequence $\left\{P_{n}^{(3)}\right\}_{n \geq 0}$, and third-order Pell-Lucas sequence $\left\{Q_{n}^{(3)}\right\}_{n \geq 0}$ are defined, respectively, by the third-order recurrence relations

$$
\begin{array}{ll}
P_{n+3}^{(3)}=2 P_{n+2}^{(3)}+P_{n+1}^{(3)}+P_{n}^{(3)}, & P_{0}^{(3)}=0, P_{1}^{(3)}=1, P_{2}^{(3)}=2 \\
Q_{n+3}^{(3)}=2 Q_{n+2}^{(3)}+Q_{n+1}^{(3)}+Q_{n}^{(3)}, & Q_{0}^{(3)}=3, Q_{1}^{(3)}=2, Q_{2}^{(3)}=6 .
\end{array}
$$

The sequences $\left\{P_{n}^{(3)}\right\}_{n \geq 0}$ and $\left\{Q_{n}^{(3)}\right\}_{n \geq 0}$ can be extended to negative subscripts by defining

$$
\begin{aligned}
& P_{-n}^{(3)}=-P_{-(n-1)}^{(3)}-2 P_{-(n-2)}^{(3)}+P_{-(n-3)}^{(3)}, \\
& Q_{-n}^{(3)}=-Q_{-(n-1)}^{(3)}-2 Q_{-(n-2)}^{(3)}+Q_{-(n-3)}^{(3)},
\end{aligned}
$$

for $n=1,2,3, \ldots$, respectively. Therefore, recurrences (3)-(4) hold for all integer $n$.

Binet formula of generalized third order Pell numbers can be given as

$$
V_{n}=\frac{b_{1} \alpha^{n}}{(\alpha-\beta)(\alpha-\gamma)}+\frac{b_{2} \beta^{n}}{(\beta-\alpha)(\beta-\gamma)}+\frac{b_{3} \gamma^{n}}{(\gamma-\alpha)(\gamma-\beta)}
$$

where

$$
b_{1}=V_{2}-(\beta+\gamma) V_{1}+\beta \gamma V_{0}, b_{2}=V_{2}-(\alpha+\gamma) V_{1}+\alpha \gamma V_{0}, b_{3}=V_{2}-(\alpha+\beta) V_{1}+\alpha \beta V_{0}
$$

Here, $\alpha, \beta$ and $\gamma$ are the roots of the cubic equation $x^{3}-2 x^{2}-x-1=0$. Moreover,

$$
\begin{aligned}
& \alpha=\frac{2}{3}+\left(\frac{61}{54}+\sqrt{\frac{29}{36}}\right)^{1 / 3}+\left(\frac{61}{54}-\sqrt{\frac{29}{36}}\right)^{1 / 3}, \\
& \beta=\frac{2}{3}+\omega\left(\frac{61}{54}+\sqrt{\frac{29}{36}}\right)^{1 / 3}+\omega^{2}\left(\frac{61}{54}-\sqrt{\frac{29}{36}}\right)^{1 / 3}, \\
& \gamma=\frac{2}{3}+\omega^{2}\left(\frac{61}{54}+\sqrt{\frac{29}{36}}\right)^{1 / 3}+\omega\left(\frac{61}{54}-\sqrt{\frac{29}{36}}\right)^{1 / 3},
\end{aligned}
$$

where

$$
\omega=\frac{-1+i \sqrt{3}}{2}=\exp (2 \pi i / 3)
$$


Note that

$$
\begin{aligned}
\alpha+\beta+\gamma & =2, \\
\alpha \beta+\alpha \gamma+\beta \gamma & =-1, \\
\alpha \beta \gamma & =1 .
\end{aligned}
$$

In the rest of the paper, for easy writing, we drop the superscripts and write $P_{n}$ and $Q_{n}$ for $P_{n}^{(3)}$ and $Q_{n}^{(3)}$, respectively. Note that $P_{n}$ is the sequence $A 077939$ in [19] associated with the expansion of $1 /\left(1-2 x-x^{2}-x^{3}\right), Q_{n}$ is the sequence $A 276225$ in [19]. For more details for the generalized third order Pell numbers, see Soykan [22].

The following Theorem presents sum formula of generalized third-order Pell numbers.

Theorem 1.1. Let $x$ be a nonzero real or complex number. For $n \geq 0$, we have the following formula: If $x^{3}+x^{2}+2 x-1 \neq 0$, then

$$
\sum_{k=0}^{n} x^{k} V_{k}=\frac{\Theta_{1}(x)}{\Theta(x)} .
$$

where

$$
\begin{aligned}
\Theta_{1}(x)= & x^{n+3} V_{n+3}-(2 x-1) x^{n+2} V_{n+2}-\left(x^{2}+2 x-1\right) x^{n+1} V_{n+1}-x^{2} V_{2} \\
& +x(2 x-1) V_{1}+\left(x^{2}+2 x-1\right) V_{0}, \\
\Theta(x)= & x^{3}+x^{2}+2 x-1 .
\end{aligned}
$$

Proof. Take $r=2, s=1, t=1$ in [20, Theorem 2.1. (a)].

The following theorem presents sum formulas of generalized third-order Pell numbers.

Theorem 1.2. For $n \geq 0$, we have the following formulas:

(a) $\sum_{i=0}^{n} V_{i}=\frac{1}{3}\left(V_{n+3}-V_{n+2}-2 V_{n+1}-V_{2}+V_{1}+2 V_{0}\right)$.

(b) $\sum_{i=0}^{n} i V_{i}=\frac{1}{9}\left((3 n+2) V_{n+3}-(3 n+5) V_{n+2}-(6 n+4) V_{n+1}+V_{2}+2 V_{1}-2 V_{0}\right)$.

(c) $\sum_{i=0}^{n} V_{i}^{2}=\frac{1}{9}\left(-V_{n+3}^{2}-10 V_{n+1}^{2}-9 V_{n+2}^{2}+2 V_{n+3} V_{n+1}+6 V_{n+3} V_{n+2}+V_{2}^{2}+9 V_{1}^{2}+10 V_{0}^{2}\right.$

$$
\left.-6 V_{2} V_{1}-2 V_{2} V_{0}\right) \text {. }
$$

(d) $\sum_{i=0}^{n} i V_{i}^{2}=\frac{1}{27}\left(-(3 n+10) V_{n+3}^{2}-(27 n+63) V_{n+2}^{2}-(30 n+40) V_{n+1}^{2}\right.$

$$
\begin{aligned}
& +2(9 n+24) V_{n+3} V_{n+2}+2(3 n+7) V_{n+3} V_{n+1}-6 V_{n+2} V_{n+1}+7 V_{2}^{2} \\
& \left.+36 V_{1}^{2}+10 V_{0}^{2}-30 V_{2} V_{1}-8 V_{2} V_{0}+6 V_{0} V_{1}\right) .
\end{aligned}
$$

Proof. (a) Take $x=1, r=2, s=1, t=1$ in [20, Theorem 2.1. (a)] or take $r=2, s=1, t=1$ in [25, Theorem 2.1. (a)].

(b) Take $x=1, r=2, s=1, t=1$ in [27, Theorem 2.1. (a)] or take $r=2, s=1, t=1$ in [29, Theorem 2.1. (a)].

(c) Take $x=1, r=2, s=1, t=1$ in [24, Theorem 3.1 (a)]. See also [23, Theorem 2.1].

(d) Take $x=1, r=2, s=1, t=1$ in [26, Theorem 2.1. (a)] or take $r=2, s=1, t=1$ in [28, Theorem 2.1. (a)]. 
Note that, using the recurrence relation $V_{n+3}=2 V_{n+2}+V_{n+1}+V_{n}$, we can write the above theorem as follows.

Theorem 1.3. For $n \geq 0$, we have the following formulas:

(a) $\sum_{i=0}^{n} V_{i}=\frac{1}{3}\left(V_{n+2}-V_{n+1}+V_{n}-V_{2}+V_{1}+2 V_{0}\right)=\frac{\Theta_{1}}{\Theta}$.

(b) $\sum_{i=0}^{n} i V_{i}=\frac{1}{9}\left((3 n-1) V_{n+2}-(3 n+2) V_{n+1}+(3 n+2) V_{n}+V_{2}+2 V_{1}-2 V_{0}\right)=\frac{\Psi_{1}}{\Psi}$.

(c) $\sum_{i=0}^{n} V_{i}^{2}=\frac{1}{9}\left(-V_{n+2}^{2}-9 V_{n+1}^{2}-V_{n}^{2}+6 V_{n+2} V_{n+1}+2 V_{n+2} V_{n}+V_{2}^{2}+9 V_{1}^{2}+10 V_{0}^{2}\right.$

$$
\left.=\frac{\Delta_{1}}{\Delta} .6 V_{2} V_{1}-2 V_{2} V_{0}\right)
$$

(d) $\sum_{i=0}^{n} i V_{i}^{2}=\frac{1}{27}\left(-(3 n+7) V_{n+2}^{2}-9(3 n+4) V_{n+1}^{2}-(3 n+10) V_{n}^{2}+6(3 n+5) V_{n+2} V_{n+1}\right.$

$$
\begin{aligned}
& +2(3 n+4) V_{n+2} V_{n}-6 V_{n+1} V_{n}+7 V_{2}^{2}+36 V_{1}^{2}+10 V_{0}^{2}-30 V_{2} V_{1} \\
& \left.\quad-8 V_{2} V_{0}+6 V_{0} V_{1}\right) \\
= & \frac{\Omega_{1}}{\Omega} .
\end{aligned}
$$

From the last Theorem, we have the following corollary which gives sum formulas of third-order Pell numbers (take $V_{n}=P_{n}$ with $P_{0}=0, P_{1}=1, P_{2}=2$ ).

Corollary 1.3.1. For $n \geq 0$, third-order Pell numbers have the following properties:

(a) $\sum_{i=0}^{n} P_{i}=\frac{1}{3}\left(P_{n+2}-P_{n+1}+P_{n}-1\right)$.

(b) $\sum_{i=0}^{n} i P_{i}=\frac{1}{9}\left((3 n-1) P_{n+2}-(3 n+2) P_{n+1}+(3 n+2) P_{n}+4\right)$.

(c) $\sum_{i=0}^{n} P_{i}^{2}=\frac{1}{9}\left(-P_{n+2}^{2}-9 P_{n+1}^{2}-P_{n}^{2}+6 P_{n+2} P_{n+1}+2 P_{n+2} P_{n}+1\right)$.

(d) $\sum_{i=0}^{n} i P_{i}^{2}=\frac{1}{27}\left(-(3 n+7) P_{n+2}^{2}-9(3 n+4) P_{n+1}^{2}-(3 n+10) P_{n}^{2}+6(3 n+5) P_{n+2} P_{n+1}\right.$

$$
\left.+2(3 n+4) P_{n+2} P_{n}-6 P_{n+1} P_{n}+4\right) .
$$

Taking $V_{n}=Q_{n}$ with $Q_{0}=3, Q_{1}=2, Q_{2}=6$ in the last Theorem, we have the following corollary which presents sum formulas of third-order Pell-Lucas numbers.

Corollary 1.3.2. For $n \geq 0$, third-order Pell-Lucas numbers have the following properties:

(a) $\sum_{i=0}^{n} Q_{i}=\frac{1}{3}\left(Q_{n+2}-Q_{n+1}+Q_{n}+2\right)$.

(b) $\sum_{i=0}^{n} i Q_{i}=\frac{1}{9}\left((3 n-1) Q_{n+2}-(3 n+2) Q_{n+1}+(3 n+2) Q_{n}+4\right)$.

(c) $\sum_{i=0}^{n} Q_{i}^{2}=\frac{1}{9}\left(-Q_{n+2}^{2}-9 Q_{n+1}^{2}-Q_{n}^{2}+6 Q_{n+2} Q_{n+1}+2 Q_{n+2} Q_{n}+54\right)$.

(d) $\sum_{i=0}^{n} i Q_{i}^{2}=\frac{1}{27}\left(-(3 n+7) Q_{n+2}^{2}-9(3 n+4) Q_{n+1}^{2}-(3 n+10) Q_{n}^{2}+6(3 n+5) Q_{n+2} Q_{n+1}\right.$

$$
\left.+2(3 n+4) Q_{n+2} Q_{n}-6 Q_{n+1} Q_{n}+18\right) .
$$




\section{Main results}

Next, we recall some information on $k$-circulant matrix and Frobenius norm, spectral norm, maximum column length norm and maximum row length norm. Let $n \geq 2$ be an integer and $k$ be any real or complex number. An $n \times n$ matrix $C_{k}=\left(c_{i j}\right) \in M_{n \times n}(\mathbb{C})$ is called a $k$-circulant matrix if it is of the form

$$
C_{k}=\left(\begin{array}{cccccc}
c_{0} & c_{1} & c_{2} & \cdots & c_{n-2} & c_{n-1} \\
k c_{n-1} & c_{0} & c_{1} & \cdots & c_{n-3} & c_{n-2} \\
k c_{n-2} & k c_{n-1} & c_{0} & \cdots & c_{n-4} & c_{n-3} \\
\vdots & \vdots & \vdots & \ddots & \vdots & \vdots \\
k c_{2} & k c_{3} & k c_{4} & \cdots & c_{0} & c_{1} \\
k c_{1} & k c_{2} & k c_{3} & \cdots & k c_{n-1} & c_{0}
\end{array}\right)_{n \times n}
$$

The $k$-circulant matrix $C_{k}$ is denoted by $C_{k}=\operatorname{Circ}_{k}\left(c_{0}, c_{1}, \ldots, c_{n-1}\right)$.

If $k=1$, then the 1-circulant matrix is called circulant matrix and denoted by $C=\operatorname{Circ}\left(c_{0}, c_{1}, \ldots, c_{n-1}\right)$. Circulant matrix was first proposed by Davis in [4]. This matrix has many interesting properties, and it is one of the most important research subject in the field of the computational and pure mathematics (see for example references given in Table 1). For instance, Deveci, Karaduman and Campbell [5] studied on the Fibonacci circulant sequences and their applications. Then, later Kızılateş and Tuglu [10] defined a new geometric circulant matrix as follows:

$$
C_{k^{*}}=\left(\begin{array}{cccccc}
c_{0} & c_{1} & c_{2} & \cdots & c_{n-2} & c_{n-1} \\
k c_{n-1} & c_{0} & c_{1} & \cdots & c_{n-3} & c_{n-2} \\
k^{2} c_{n-2} & k c_{n-1} & c_{0} & \cdots & c_{n-4} & c_{n-3} \\
\vdots & \vdots & \vdots & \ddots & \vdots & \vdots \\
k^{n-1} c_{1} & k^{n-2} c_{2} & k^{n-3} c_{3} & \cdots & k c_{n-1} & c_{0}
\end{array}\right)_{n \times n}
$$

and then they obtained the bounds for the spectral norms of geometric circulant matrices with the generalized Fibonacci and Lucas numbers. When the parameter satisfies $k=1$, we get the classical circulant matrix. See also Polatl [14] for the spectral norms of $k$-circulant matrices with a type of Catalan triangle numbers.

The Frobenius (or Euclidean) norm and spectral norm of an $m \times n$ matrix $A=\left(a_{i j}\right)_{m \times n} \in$ $M_{m \times n}(\mathbb{C})$ are defined respectively as follows:

$$
\|A\|_{F}=\left(\sum_{i=1}^{m} \sum_{j=1}^{n}\left|a_{i j}\right|^{2}\right)^{1 / 2} \text { and }\|A\|_{2}=\left(\max _{1 \leq i \leq n}\left|\lambda_{i}\left(A^{*} A\right)\right|\right)^{1 / 2}
$$

where $\lambda_{i}\left(A^{*} A\right)$ 's are the eigenvalues of the matrix $A^{*} A$ and $A^{*}$ is the conjugate of transpose of the matrix $A$. The following inequality holds for any matrix $A=\left(a_{i j}\right)_{m \times n} \in M_{n \times n}(\mathbb{C})$ (see [35, Theorem 1 and Table 1]):

$$
\frac{1}{\sqrt{n}}\|A\|_{F} \leq\|A\|_{2} \leq\|A\|_{F}
$$

It follows that

$$
\|A\|_{2} \leq\|A\|_{F} \leq \sqrt{n}\|A\|_{2} .
$$


In literature there are other types of norms of matrices. The maximum column sum matrix norm of an $n \times n$ matrix $A=\left(a_{i j}\right)$ is $\|A\|_{1}=\max _{1 \leq j \leq n} \sum_{i=1}^{n}\left|a_{i j}\right|$ and the maximum row sum matrix norm is $\|A\|_{\infty}=\max _{1 \leq i \leq n} \sum_{j=1}^{n}\left|a_{i j}\right|$. The maximum column length norm $c_{1}(A)$ and the maximum row length norm $r_{1}(A)$ of an $m \times n$ matrix $A=\left(a_{i j}\right)$ are defined as follows:

$$
c_{1}(A)=\max _{1 \leq j \leq n}\left(\sum_{i=1}^{n}\left|a_{i j}\right|^{2}\right)^{1 / 2} \text { and } r_{1}(A)=\max _{1 \leq i \leq n}\left(\sum_{j=1}^{n}\left|a_{i j}\right|^{2}\right)^{1 / 2} .
$$

There is a relation between $\|.\|_{2}, c_{1}($.$) and r_{1}($.$) norms:$

Lemma 2.1. [8] For any matrices $A=\left(a_{i j}\right)_{m \times n} \in M_{m \times n}(\mathbb{C})$ and $B=\left(b_{i j}\right)_{m \times n} \in M_{m \times n}(\mathbb{C})$, we have

$$
\|A \circ B\|_{2} \leq r_{1}(A) c_{1}(B)
$$

and

$$
\|A \circ B\|_{2} \leq\|A\|_{2}\|B\|_{2}
$$

and

$$
\|A \otimes B\|_{2}=\|A\|_{2}\|B\|_{2}
$$

where $A \circ B$ is the Hadamard product which is defined by

$$
A \circ B=\left(a_{i j} b_{i j}\right),
$$

$A \otimes B$ is the Kronecker product which is defined by

$$
A \otimes B=\left(a_{i j} B\right)
$$

Calculations of the above norms $\|A\|_{F},\|A\|_{2}, c_{1}(A)$ and $r_{1}(A)$ require the sum of the squares of the numbers $a_{i j}$. As in our case, the numbers $a_{i j}$ can be chosen as elements of generalized third-order Pell sequence. For more details on norm of matrices, see for example [7].

In the following Table 1, we present a few special study on the Frobenius norm, spectral norm, maximum column length norm and maximum row length norm of circulant $(k$-circulant, geometric circulant, semicirculant) matrices with the generalized $m$-step Fibonacci sequences which require sum formulas of second powers of numbers in $m$-step Fibonacci sequences $(m=2,3,4)$.

\begin{tabular}{|c|c|c|}
\hline Order & Name of sequence & Papers \\
\hline \multirow{3}{*}{ Second order } & Fibonacci, Lucas & {$[5,6,10,30]$} \\
\cline { 2 - 3 } & Pell, Pell-Lucas & {$[1,31]$} \\
\cline { 2 - 3 } & Jacobsthal, Jacobsthal-Lucas & {$[15,32,33,34]$} \\
\hline \multirow{2}{*}{ Third order } & Tribonacci, Tribonacci-Lucas & {$[9,16,17]$} \\
\cline { 2 - 3 } & Padovan, Perrin & {$[3,12,18]$} \\
\hline Fourth order & Tetranacci, Tetranacci-Lucas & {$[11]$} \\
\hline
\end{tabular}

Table 1. Papers on the norms

We need the following two lemmas for our calculations. 
Lemma 2.2. [2, Lemma 4] Let $C_{k}=\operatorname{Circ}_{k}\left(c_{0}, c_{1}, \ldots, c_{n-1}\right)$ be a $n \times n k$-circulant matrix. Then we have

$$
\lambda_{j}\left(C_{k}\right)=\sum_{p=0}^{n-1} k^{\frac{p}{n}} \omega^{-j p} c_{p}=\sum_{p=0}^{n-1}\left(k^{\frac{1}{n}} \omega^{-j}\right)^{p} c_{p}
$$

where $\omega=\exp (2 \pi i / n)=e^{\frac{2 \pi i}{n}}, j=0,1,2, \ldots, n-1$. Moreover, in this case

$$
c_{p}=\frac{1}{n} \sum_{j=0}^{n-1}\left(k^{\frac{1}{n}} \omega^{-j}\right)^{-p} \lambda_{j}\left(C_{k}\right), \quad p=0,1,2, \ldots, n-1 .
$$

Lemma 2.3. [7] Let $A$ be a $n \times n$ matrix with eigenvalues $\lambda_{1}, \lambda_{2}, \lambda_{3}, \ldots, \lambda_{n}$. Then, $A$ is a normal matrix if and only if the eigenvalues of $A A^{*}$ are $\left|\lambda_{1}\right|^{2},\left|\lambda_{2}\right|^{2},\left|\lambda_{3}\right|^{2}, \ldots,\left|\lambda_{n}\right|^{2}$ where $A^{*}$ is the conjugate of transpose of the matrix $A$.

Next, we define $k$-circulant matrix with generalized third-order Pell numbers entries. Throughout this paper, the $k$-circulant matrix, whose entries are the generalized third-order Pell numbers, will be denoted by $C_{n}(V)_{k}=\operatorname{Circ}_{k}\left(V_{0}, V_{1}, \ldots, V_{n-1}\right)$ :

Definition 1. A $n \times n k$-circulant matrix with generalized third-order Pell numbers entries is defined by

$$
C_{n}(V)_{k}=\operatorname{Circ}_{k}\left(V_{0}, V_{1}, \ldots, V_{n-1}\right)=\left(\begin{array}{cccccc}
V_{0} & V_{1} & V_{2} & \cdots & V_{n-2} & V_{n-1} \\
k V_{n-1} & V_{0} & V_{1} & \cdots & V_{n-3} & V_{n-2} \\
k V_{n-2} & k V_{n-1} & V_{0} & \cdots & V_{n-4} & V_{n-3} \\
\vdots & \vdots & \vdots & \ddots & \vdots & \vdots \\
k V_{1} & k V_{2} & k V_{3} & \cdots & k V_{n-1} & V_{0}
\end{array}\right)_{n \times n} .
$$

We call this matrix generalized third-order Pell k-circulant matrix. We consider two special cases of generalized third-order Pell $k$-circulant matrix, namely third-order Pell $k$-circulant matrix: $C_{n}(P)_{k}=\operatorname{Circ}_{k}\left(P_{0}, P_{1}, \ldots, P_{n-1}\right)$ and third-order Pell-Lucas $k$-circulant matrix: $C_{n}(Q)_{k}=$ $\operatorname{Circ}_{k}\left(Q_{0}, Q_{1}, \ldots, Q_{n-1}\right)$. We denote the sum of entries of $C_{n}(V)_{k}$ as $S\left(C_{n}(V)_{k}\right)$.

Lemma 2.4. The sum of entries of $C_{n}(V)_{k}$ is

$$
\begin{aligned}
S\left(C_{n}(V)_{k}\right)= & \frac{1}{9}\left((-k+3 k n+1) V_{n+2}-(2 k+3 k n-2) V_{n+1}-2(-k+3 k n+1) V_{n}\right. \\
& \left.+(k-3 n-1) V_{2}+(2 k+3 n-2) V_{1}-2(k-3 n-1) V_{0}\right) .
\end{aligned}
$$

Proof. From the definition of $C_{n}(V)_{k}$, using Theorem 1.3, we obtain

$$
\begin{aligned}
S\left(C_{n}(V)_{k}\right)= & n V_{0}+((n-1)+k) V_{1}+((n-2)+2 k) V_{2}+\cdots+(1+(n-1) k) V_{n-1} \\
= & \sum_{i=0}^{n-1}(n-i) V_{i}+k \sum_{i=1}^{n-1} i V_{i} \\
= & n \sum_{i=0}^{n-1} V_{i}+(k-1) \sum_{i=1}^{n-1} i V_{i} \\
= & n\left(-V_{n}+\sum_{i=0}^{n} V_{i}\right)+(k-1)\left(-n V_{n}+\sum_{i=0}^{n} i V_{i}\right) \\
= & \frac{1}{9}\left((-k+3 k n+1) V_{n+2}-(2 k+3 k n-2) V_{n+1}-2(-k+3 k n+1) V_{n}\right. \\
& \left.+(k-3 n-1) V_{2}+(2 k+3 n-2) V_{1}-2(k-3 n-1) V_{0}\right) .
\end{aligned}
$$


Taking $V_{n}=P_{n}$ with $P_{0}=0, P_{1}=1, P_{2}=2$ and $V_{n}=Q_{n}$ with $Q_{0}=3, Q_{1}=2, Q_{2}=6$, respectively, in the last Lemma, we obtain the following corollary.

Corollary 2.4.1. We have the following results:

(a) The sum of entries of $C_{n}(P)_{k}$ is

$$
\begin{aligned}
S\left(C_{n}(P)_{k}\right)= & \frac{1}{9}\left((-k+3 k n+1) P_{n+2}-(2 k+3 k n-2) P_{n+1}-2(-k+3 k n+1) P_{n}\right. \\
& +(4 k-3 n-4)) .
\end{aligned}
$$

(b) The sum of entries of $C_{n}(Q)_{k}$ is

$$
\begin{aligned}
S\left(C_{n}(Q)_{k}\right)= & \frac{1}{9}\left((-k+3 k n+1) Q_{n+2}-(2 k+3 k n-2) Q_{n+1}-2(-k+3 k n+1) Q_{n}\right. \\
& +(4 k+6 n-4)) .
\end{aligned}
$$

Next, we present the maximum column sum matrix norm $\left\|C_{n}(V)_{k}\right\|_{1}$ and the maximum row sum matrix norm $\left\|C_{n}(V)_{k}\right\|_{\infty}$ of the matrix $C_{n}(V)_{k}=\left(c_{i j}\right)$ under certain condition on the generalized third-order Pell sequence $V_{n}$ and $k$.

Theorem 2.5. Suppose that $V_{p} \geq 0$ for all the nonnegative integers $p$. Then we have the following formulas: If $k \geq 1$ then

$$
\left\|C_{n}(V)_{k}\right\|_{1}=\left\|C_{n}(V)_{k}\right\|_{\infty}=\frac{1}{3}\left(k V_{n+2}-k V_{n+1}-2 k V_{n}-k V_{2}+k V_{1}+(3-k) V_{0}\right),
$$

and if $k<1$, then

$$
\left\|C_{n}(V)_{k}\right\|_{1}=\left\|C_{n}(V)_{k}\right\|_{\infty}=\frac{1}{3}\left(V_{n+2}-V_{n+1}-2 V_{n}-V_{2}+V_{1}+2 V_{0}\right) .
$$

Proof. Suppose that $k \geq 1$. Then from the definition of the matrix $C_{n}(V)_{k}=\left(c_{i j}\right)$, using Theorem 1.3, we can write

$$
\begin{aligned}
\left\|C_{n}(V)_{k}\right\|_{1} & =\max _{1 \leq j \leq n} \sum_{i=1}^{n}\left|c_{i j}\right|=\max _{1 \leq j \leq n}\left\{\left|c_{1 j}\right|+\left|c_{2 j}\right|+\left|c_{3 j}\right|+\cdots+\left|c_{n j}\right|\right\} \\
& =\left|c_{11}\right|+\left|c_{21}\right|+\left|c_{31}\right|+\cdots+\left|c_{n 1}\right| \\
& =V_{0}+k V_{n-1}+k V_{n-2}+\cdots+k V_{3}+k V_{2}+k V_{1} \\
& =\left(V_{0}-k V_{0}-k V_{n}\right)+k \sum_{i=0}^{n} V_{i} \\
& =\frac{1}{3}\left(k V_{n+2}-k V_{n+1}-2 k V_{n}-k V_{2}+k V_{1}+(3-k) V_{0}\right) .
\end{aligned}
$$

Similarly, we have

$$
\left\|C_{n}(V)_{k}\right\|_{\infty}=\frac{1}{3}\left(k V_{n+2}-k V_{n+1}-2 k V_{n}-k V_{2}+k V_{1}+(3-k) V_{0}\right) .
$$

Suppose now that $k<1$. Then from the definition of the matrix $C_{n}(V)_{k}=\left(c_{i j}\right)$, using Theorem 1.3, we can write 


$$
\begin{aligned}
\left\|C_{n}(V)_{k}\right\|_{1} & =\max _{1 \leq j \leq n} \sum_{i=1}^{n}\left|c_{i j}\right|=\max _{1 \leq j \leq n}\left\{\left|c_{1 j}\right|+\left|c_{2 j}\right|+\left|c_{3 j}\right|+\cdots+\left|c_{n j}\right|\right\} \\
& =\left|c_{1 n}\right|+\left|c_{2 n}\right|+\left|c_{3 n}\right|+\cdots+\left|c_{n n}\right| \\
& =V_{n-1}+V_{n-2}+\cdots+V_{3}+V_{2}+V_{1}+V_{0} \\
& =-V_{n}+\sum_{i=0}^{n} V_{i} \\
& =\frac{1}{3}\left(V_{n+2}-V_{n+1}-2 V_{n}-V_{2}+V_{1}+2 V_{0}\right) .
\end{aligned}
$$

Similarly, we have

$$
\left\|C_{n}(V)_{k}\right\|_{\infty}=\frac{1}{3}\left(V_{n+2}-V_{n+1}-2 V_{n}-V_{2}+V_{1}+2 V_{0}\right) .
$$

Taking $V_{n}=P_{n}$ with $P_{0}=0, P_{1}=1, P_{2}=2$ and $V_{n}=Q_{n}$ with $Q_{0}=3, Q_{1}=2, Q_{2}=6$, respectively in the last theorem, we obtain the following corollary.

Corollary 2.5.1. We have the following results:

(a) If $k \geq 1$, then

$$
\left\|C_{n}(P)_{k}\right\|_{1}=\left\|C_{n}(P)_{k}\right\|_{\infty}=\frac{k}{3}\left(P_{n+2}-P_{n+1}-2 P_{n}-1\right),
$$

and if $k<1$, then

$$
\left\|C_{n}(P)_{k}\right\|_{1}=\left\|C_{n}(P)_{k}\right\|_{\infty}=\frac{1}{3}\left(P_{n+2}-P_{n+1}-2 P_{n}-1\right) .
$$

(b) If $k \geq 1$, then

$$
\left\|C_{n}(Q)_{k}\right\|_{1}=\left\|C_{n}(Q)_{k}\right\|_{\infty}=\frac{1}{3}\left(k Q_{n+2}-k Q_{n+1}-2 k Q_{n}+(9-7 k)\right),
$$

and if $k<1$, then

$$
\left\|C_{n}(Q)_{k}\right\|_{1}=\left\|C_{n}(Q)_{k}\right\|_{\infty}=\frac{1}{3}\left(Q_{n+2}-Q_{n+1}-2 Q_{n}+2\right) .
$$

Now, we determine the Euclidean (Frobenius) norm of $k$-circulant matrix $C_{n}(V)_{k}$.

Theorem 2.6. The Euclidean (Frobenius) norm of $k$-circulant matrix $C_{n}(V)_{k}$ is:

$$
\left\|C_{n}(V)_{k}\right\|_{F}=\sqrt{n\left(\varphi_{1}(V)\right)+\varphi_{2}(V)}
$$

where

$$
\begin{aligned}
\varphi_{1}(V)=\frac{1}{9} & \left(-V_{n+2}^{2}-9 V_{n+1}^{2}-10 V_{n}^{2}+6 V_{n+2} V_{n+1}+2 V_{n+2} V_{n}+V_{2}^{2}+9 V_{1}^{2}+10 V_{0}^{2}\right. \\
& \left.\quad-6 V_{2} V_{1}-2 V_{2} V_{0}\right), \\
\varphi_{2}(V)=\frac{1}{27} & \left(|k|^{2}-1\right)\left(-(3 n+7) V_{n+2}^{2}-9(3 n+4) V_{n+1}^{2}-10(3 n+1) V_{n}^{2}+6(3 n+5) V_{n+2} V_{n+1}\right. \\
& \left.+2(3 n+4) V_{n+2} V_{n}-6 V_{n+1} V_{n}+7 V_{2}^{2}+36 V_{1}^{2}+10 V_{0}^{2}-30 V_{2} V_{1}-8 V_{2} V_{0}+6 V_{0} V_{1}\right) .
\end{aligned}
$$


Proof. From the definition of the Euclidean norm of a matrix, using Theorem 1.3, we obtain

$$
\begin{aligned}
\left(\left\|C_{n}(V)_{k}\right\|_{F}\right)^{2} & =\sum_{i=1, j=1}^{n}\left|c_{i j}\right|^{2} \\
& =\sum_{i=0}^{n-1}(n-i) V_{i}^{2}+|k|^{2} \sum_{i=1}^{n-1} i V_{i}^{2} \\
& =n \sum_{i=0}^{n-1} V_{i}^{2}+\left(|k|^{2}-1\right) \sum_{i=1}^{n-1} i V_{i}^{2} \\
& =n\left(\varphi_{1}(V)\right)+\varphi_{2}(V)
\end{aligned}
$$

where $\varphi_{1}(V)$ and $\varphi_{2}(V)$ are as in the statement of the theorem. Now, it follows that

$$
\left\|C_{n}(V)_{k}\right\|_{F}=\sqrt{n\left(\varphi_{1}(V)\right)+\varphi_{2}(V)} .
$$

Note that

$$
\varphi_{1}(V)=\sum_{i=0}^{n-1} V_{i}^{2} \quad \text { and } \quad \varphi_{2}(V)=\left(|k|^{2}-1\right) \sum_{i=1}^{n-1} i V_{i}^{2} .
$$

Taking $V_{n}=P_{n}$ with $P_{0}=0, P_{1}=1, P_{2}=2$ and $V_{n}=Q_{n}$ with $Q_{0}=3, Q_{1}=2, Q_{2}=6$, respectively in the last Theorem, we obtain the following corollary.

Corollary 2.6.1. We have the following results:

(a) The Euclidean (Frobenius) norm of $k$-circulant matrix $C_{n}(P)_{k}$ is:

$$
\left\|C_{n}(P)_{k}\right\|_{F}=\sqrt{n\left(\varphi_{1}(P)\right)+\varphi_{2}(P)}
$$

where

$$
\begin{aligned}
\varphi_{1}(P)= & \frac{1}{9}\left(-P_{n+2}^{2}-9 P_{n+1}^{2}-10 P_{n}^{2}+6 P_{n+2} P_{n+1}+2 P_{n+2} P_{n}+1\right) \\
\varphi_{2}(P)= & \frac{1}{27}\left(|k|^{2}-1\right)\left(-(3 n+7) P_{n+2}^{2}-9(3 n+4) P_{n+1}^{2}-10(3 n+1) P_{n}^{2}\right. \\
& \left.\quad+6(3 n+5) P_{n+2} P_{n+1}+2(3 n+4) P_{n+2} P_{n}-6 P_{n+1} P_{n}+4\right) .
\end{aligned}
$$

(b) The Euclidean (Frobenius) norm of k-circulant matrix $C_{n}(Q)_{k}$ is:

$$
\left\|C_{n}(Q)_{k}\right\|_{F}=\sqrt{n\left(\varphi_{1}(Q)\right)+\varphi_{2}(Q)}
$$

where

$$
\begin{aligned}
\varphi_{1}(Q)= & \frac{1}{9}\left(-Q_{n+2}^{2}-9 Q_{n+1}^{2}-10 Q_{n}^{2}+6 Q_{n+2} Q_{n+1}+2 Q_{n+2} Q_{n}+54\right) \\
\varphi_{2}(Q)=\frac{1}{27}\left(|k|^{2}-1\right)( & -(3 n+7) Q_{n+2}^{2}-9(3 n+4) Q_{n+1}^{2}-10(3 n+1) Q_{n}^{2} \\
& \left.+6(3 n+5) Q_{n+2} Q_{n+1}+2(3 n+4) Q_{n+2} Q_{n}-6 Q_{n+1} Q_{n}+18\right) .
\end{aligned}
$$

The following theorem gives us the eigenvalues of the matrix in (7). 
Theorem 2.7. The eigenvalues of $C_{n}(V)_{k}$ are

$$
\lambda_{j}\left(C_{n}(V)\right)=\frac{\Phi_{j}(V)}{\left(k^{\frac{1}{n}} \omega^{-j}\right)^{3}+\left(k^{\frac{1}{n}} \omega^{-j}\right)^{2}+2\left(k^{\frac{1}{n}} \omega^{-j}\right)-1}
$$

where

$$
\begin{aligned}
\Phi_{j}(V)=k V_{n}-V_{0} & -k^{\frac{1}{n}}\left(-k V_{n+1}+2 k V_{n}+V_{1}-2 V_{0}\right) \omega^{-j} \\
& +k^{\frac{2}{n}}\left(k V_{n+2}-2 k V_{n+1}-k V_{n}-V_{2}+2 V_{1}+V_{0}\right) \omega^{-2 j}
\end{aligned}
$$

and

$$
\begin{aligned}
\omega & =\exp (2 \pi i / n)=e^{\frac{2 \pi i}{n}}, \\
j & =0,1,2,3, \ldots, n-1 .
\end{aligned}
$$

Proof. By using Lemma 2.2, we obtain

$$
\begin{aligned}
\lambda_{j}\left(C_{n}(V)_{k}\right) & =\sum_{p=0}^{n-1} k^{\frac{p}{n}} \omega^{-j p} V_{p} \\
& =-k \omega^{-j n} V_{n}+\sum_{p=0}^{n} k^{\frac{p}{n}} \omega^{-j p} V_{p} \\
& =-k \omega^{-j n} V_{n}+\sum_{p=0}^{n}\left(k^{\frac{1}{n}} \omega^{-j}\right)^{p} V_{p} .
\end{aligned}
$$

Now using Theorem 1.1 (by putting $x=k^{\frac{1}{n}} \omega^{-j}$ ) and recurrence relation

$$
V_{n+3}=2 V_{n+2}+V_{n+1}+V_{n}
$$

we obtain required result.

Taking $V_{n}=P_{n}$ with $P_{0}=0, P_{1}=1, P_{2}=2$ and $V_{n}=Q_{n}$ with $Q_{0}=3, Q_{1}=2, Q_{2}=6$, respectively, in the last Theorem, we obtain the following corollary.

Corollary 2.7.1. We have the following results:

(a) The eigenvalues of $C_{n}(P)_{k}$ are

$$
\lambda_{j}\left(C_{n}(P)\right)=\frac{\Phi_{j}(P)}{\left(k^{\frac{1}{n}} \omega^{-j}\right)^{3}+\left(k^{\frac{1}{n}} \omega^{-j}\right)^{2}+2\left(k^{\frac{1}{n}} \omega^{-j}\right)-1},
$$

(b) the eigenvalues of $C_{n}(Q)_{k}$ are

$$
\lambda_{j}\left(C_{n}(Q)\right)=\frac{\Phi_{j}(Q)}{\left(k^{\frac{1}{n}} \omega^{-j}\right)^{3}+\left(k^{\frac{1}{n}} \omega^{-j}\right)^{2}+2\left(k^{\frac{1}{n}} \omega^{-j}\right)-1},
$$

where

$$
\begin{aligned}
\Phi_{j}(P) & =k P_{n}-k^{\frac{1}{n}}\left(-k P_{n+1}+2 k P_{n}+1\right) \omega^{-j}+k^{\frac{2}{n}}\left(k P_{n+2}-2 k P_{n+1}-k P_{n}\right) \omega^{-2 j}, \\
\Phi_{j}(Q) & =k Q_{n}-3-k^{\frac{1}{n}}\left(-k Q_{n+1}+2 k Q_{n}-4\right) \omega^{-j}+k^{\frac{2}{n}}\left(k Q_{n+2}-2 k Q_{n+1}-k Q_{n}+1\right) \omega^{-2 j}, \\
\omega & =\exp (2 \pi i / n)=e^{\frac{2 \pi i}{n}}, \quad j=0,1,2,3, \ldots, n-1 .
\end{aligned}
$$

The following theorem presents the upper and lower bounds of the spectral norm of $C_{n}(V)_{k}$. 
Theorem 2.8. Let $C_{n}(V)_{k}=\operatorname{Circ}_{k}\left(V_{0}, V_{1}, \ldots, V_{n-1}\right)$ be a $k$-circulant matrix. Then if $|k| \geq 1$ then

$$
\sqrt{\varphi_{1}(V)} \leq\left\|C_{n}(V)_{k}\right\|_{2} \leq \sqrt{V_{0}^{2}+|k|^{2}\left(-V_{0}^{2}+\varphi_{1}(V)\right)} \sqrt{1-V_{0}^{2}+\varphi_{1}(V)}
$$

and if $|k|<1$ then

$$
|k| \sqrt{\varphi_{1}(V)} \leq\left\|C_{n}(V)_{k}\right\|_{2} \leq \sqrt{n\left(\varphi_{1}(V)\right)}
$$

where $\varphi_{1}(V)$ is as in Theorem 2.6.

Proof. Note that we can write $\varphi_{1}(V)$ as in the following forms.

$$
\begin{aligned}
\varphi_{1}(V)= & \sum_{i=0}^{n-1} V_{i}^{2} \\
= & \frac{1}{9}\left(-V_{n+2}^{2}-9 V_{n+1}^{2}-10 V_{n}^{2}+6 V_{n+2} V_{n+1}+2 V_{n+2} V_{n}+V_{2}^{2}+9 V_{1}^{2}+10 V_{0}^{2}\right. \\
& \left.\quad-6 V_{2} V_{1}-2 V_{2} V_{0}\right), \\
\varphi_{1}(V)= & V_{0}^{2}+\sum_{i=1}^{n-1} V_{i}^{2} \Rightarrow-V_{0}^{2}+\varphi_{1}(V)=\sum_{i=1}^{n-1} V_{i}^{2} .
\end{aligned}
$$

From Theorem 2.6, we know that the Euclidean (Frobenius) norm of $k$-circulant matrix $C_{n}(V)_{k}$ is

$$
\begin{aligned}
\left(\left\|C_{n}(V)_{k}\right\|_{F}\right)^{2} & =\sum_{i=0}^{n-1}(n-i) V_{i}^{2}+|k|^{2} \sum_{i=1}^{n-1} i V_{i}^{2} \\
& =n \sum_{i=0}^{n-1} V_{i}^{2}+\left(|k|^{2}-1\right) \sum_{i=1}^{n-1} i V_{i}^{2} .
\end{aligned}
$$

If $|k| \geq 1$, then we get, using Theorem 1.3,

$$
\left(\left\|C_{n}(V)_{k}\right\|_{F}\right)^{2} \geq \sum_{i=0}^{n-1}(n-i) V_{i}^{2}+\sum_{i=1}^{n-1} i V_{i}^{2}=n \sum_{i=0}^{n-1} V_{i}^{2}=n\left(\varphi_{1}(V)\right)
$$

i.e., $\left\|C_{n}(V)_{k}\right\|_{F} \geq \sqrt{n\left(\varphi_{1}(V)\right)}$. It follows that

$$
\frac{\left\|C_{n}(V)_{k}\right\|_{F}}{\sqrt{n}} \geq \sqrt{\varphi_{1}(V)}
$$

Then by (6), we obtain

$$
\left\|C_{n}(V)_{k}\right\|_{2} \geq \sqrt{\varphi_{1}(V)}
$$

Similarly, If $|k|<1$, then we obtain

$$
\begin{aligned}
\left\|C_{n}(V)_{k}\right\|_{F}^{2} & =\sum_{i=0}^{n-1}(n-i) V_{i}^{2}+|k|^{2} \sum_{i=1}^{n-1} i V_{i}^{2} \\
& \geq \sum_{i=0}^{n-1}(n-i)|k|^{2} V_{i}^{2}+|k|^{2} \sum_{i=1}^{n-1} i V_{i}^{2}=n|k|^{2} \sum_{i=0}^{n-1} V_{i}^{2} \\
& =n|k|^{2}\left(\varphi_{1}(V)\right) .
\end{aligned}
$$

i.e., $\left\|C_{n}(V)_{k}\right\|_{F} \geq \sqrt{n|k|^{2}\left(\varphi_{1}(V)\right)}$. It follows that

$$
\frac{\left\|C_{n}(V)_{k}\right\|_{F}}{\sqrt{n}} \geq|k| \sqrt{\varphi_{1}(V)} .
$$


Then by considering (6), we get

$$
\left\|C_{n}(V)_{k}\right\|_{2} \geq|k| \sqrt{\left(\varphi_{1}(V)\right)} .
$$

Now, for $|k| \geq 1$, we give the upper bound for the spectral norm of the matrix $C_{n}(V)_{k}$ as follows. Let the matrices $B$ and $C$ be as

$$
B=\left(\begin{array}{cccccc}
V_{0} & 1 & 1 & \cdots & 1 & 1 \\
k V_{n-1} & V_{0} & 1 & \cdots & 1 & 1 \\
k V_{n-2} & k V_{n-1} & V_{0} & \cdots & 1 & 1 \\
\vdots & \vdots & \vdots & \ddots & \vdots & \vdots \\
k V_{1} & k V_{2} & k V_{3} & \cdots & k V_{n-1} & V_{0}
\end{array}\right)_{n \times n}
$$

and

$$
C=\left(\begin{array}{cccccc}
1 & V_{1} & V_{2} & \cdots & V_{n-2} & V_{n-1} \\
1 & 1 & V_{1} & \cdots & V_{n-3} & V_{n-2} \\
1 & 1 & 1 & \cdots & V_{n-4} & V_{n-3} \\
\vdots & \vdots & \vdots & \ddots & \vdots & \vdots \\
1 & 1 & 1 & \cdots & 1 & 1
\end{array}\right)_{n \times n}
$$

such that $C_{n}(V)_{k}=B \circ C$. Then we obtain

$$
\begin{aligned}
& r_{1}(B)=\max _{1 \leq i \leq n}\left(\sum_{j=1}^{n}\left|b_{i j}\right|^{2}\right)^{1 / 2}=\sqrt{V_{0}^{2}+|k|^{2} \sum_{j=1}^{n-1} V_{j}^{2}}=\sqrt{V_{0}^{2}+|k|^{2}\left(-V_{0}^{2}+\varphi_{1}(V)\right)}, \\
& c_{1}(C)=\max _{1 \leq j \leq n}\left(\sum_{i=1}^{n}\left|c_{i j}\right|^{2}\right)^{1 / 2}=\sqrt{1+\sum_{i=1}^{n-1} V_{i}^{2}}=\sqrt{1-V_{0}^{2}+\varphi_{1}(V)} .
\end{aligned}
$$

By Lemma 2.1, we have

$$
\left\|C_{n}(V)_{k}\right\|_{2} \leq r_{1}(B) c_{1}(C)=\sqrt{V_{0}^{2}+|k|^{2}\left(-V_{0}^{2}+\varphi_{1}(V)\right)} \sqrt{1-V_{0}^{2}+\varphi_{1}(V)} .
$$

For $|k|<1$, we give the upper bound for the spectral norm of the matrix $C_{n}(V)_{k}$ as follows. We define the matrices $D$ and $E$ as

$$
D=\left(\begin{array}{cccccc}
1 & 1 & 1 & \cdots & 1 & 1 \\
k & 1 & 1 & \cdots & 1 & 1 \\
k & k & 1 & \cdots & 1 & 1 \\
\vdots & \vdots & \vdots & \ddots & \vdots & \vdots \\
k & k & k & \cdots & k & 1
\end{array}\right)_{n \times n}
$$

and

such that $C_{n}(V)_{k}=D \circ E$.

$$
E=\left(\begin{array}{cccccc}
V_{0} & V_{1} & V_{2} & \cdots & V_{n-2} & V_{n-1} \\
V_{n-1} & V_{0} & V_{1} & \cdots & V_{n-3} & V_{n-2} \\
V_{n-2} & V_{n-1} & V_{0} & \cdots & V_{n-4} & V_{n-3} \\
\vdots & \vdots & \vdots & \ddots & \vdots & \vdots \\
V_{1} & V_{2} & V_{3} & \cdots & V_{n-1} & V_{0}
\end{array}\right)_{n \times n}
$$


Then we obtain

$$
r_{1}(D)=\max _{1 \leq i \leq n}\left(\sum_{j=1}^{n}\left|d_{i j}\right|^{2}\right)^{1 / 2}=\sqrt{n}
$$

and

$$
c_{1}(E)=\max _{1 \leq j \leq n}\left(\sum_{i=1}^{n}\left|e_{i j}\right|^{2}\right)^{1 / 2}=\sqrt{\sum_{i=0}^{n-1} V_{i}^{2}}=\sqrt{\varphi_{1}(V)} .
$$

By Lemma 2.1, we have

$$
\left\|C_{n}(V)_{k}\right\|_{2} \leq r_{1}(D) c_{1}(E)=\sqrt{n\left(\varphi_{1}(V)\right)}
$$

This completes the proof.

We consider two special cases of the above theorem: the upper and lower bounds of the spectral norm of third-order Pell $k$-circulant matrix: $C_{n}(P)_{k}=\operatorname{Circ}_{k}\left(P_{0}, P_{1}, \ldots, P_{n-1}\right)$ and the upper and lower bounds of the spectral norm of third-order Pell-Lucas $k$-circulant matrix: $C_{n}(Q)_{k}=\operatorname{Circ}_{k}\left(Q_{0}, Q_{1}, \ldots, Q_{n-1}\right)$.

Firstly, the following corollary gives the upper and lower bounds of the spectral norm of $C_{n}(P)_{k}$.

Corollary 2.8.1. Let $C_{n}(P)_{k}=\operatorname{Circ}_{k}\left(P_{0}, P_{1}, \ldots, P_{n-1}\right)$ be third-order Pell $k$-circulant matrix. Then if $|k| \geq 1$, then

$$
\sqrt{\varphi_{1}(P)} \leq\left\|C_{n}(P)_{k}\right\|_{2} \leq \sqrt{P_{0}^{2}+|k|^{2}\left(-P_{0}^{2}+\varphi_{1}(P)\right)} \sqrt{1-P_{0}^{2}+\varphi_{1}(P)}
$$

and if $|k|<1$, then

$$
|k| \sqrt{\varphi_{1}(P)} \leq\left\|C_{n}(P)_{k}\right\|_{2} \leq \sqrt{n\left(\varphi_{1}(P)\right)},
$$

where $\varphi_{1}(P)$ is as in Corollary 2.6.1.

Proof. Take $V_{n}=P_{n}, P_{0}=0, P_{1}=1, P_{2}=2$ in Theorem 2.8 .

Secondly, the following corollary gives the upper and lower bounds of the spectral norm of $C_{n}(Q)_{k}$

Corollary 2.8.2. Let $C_{n}(Q)_{k}=\operatorname{Circ}_{k}\left(Q_{0}, Q_{1}, \ldots, Q_{n-1}\right)$ be a third-order Pell-Lucas $k$-circulant matrix. Then if $|k| \geq 1$, then

$$
\sqrt{\varphi_{1}(Q)} \leq\left\|C_{n}(Q)_{k}\right\|_{2} \leq \sqrt{Q_{0}^{2}+|k|^{2}\left(-Q_{0}^{2}+\varphi_{1}(Q)\right)} \sqrt{1-Q_{0}^{2}+\varphi_{1}(Q)}
$$

and if $|k|<1$, then

$$
|k| \sqrt{\varphi_{1}(Q)} \leq\left\|C_{n}(Q)_{k}\right\|_{2} \leq \sqrt{n\left(\varphi_{1}(Q)\right)},
$$

where $\varphi_{1}(Q)$ is as in Corollary 2.6.1.

Proof. Take $V_{n}=Q_{n}, Q_{0}=3, Q_{1}=2, Q_{2}=6$ in Theorem 2.8 .

Next, we present the determinant of $C_{n}(V)_{k}$. 
Theorem 2.9. The determinant of $C_{n}(V)_{k}$ is given by

$$
\operatorname{det}\left(C_{n}(V)_{k}\right)=\frac{\Lambda_{1}^{n}\left(1-\left(\frac{\Lambda_{2}-\sqrt{\Lambda_{2}^{2}-4 \Lambda_{1} \Lambda_{3}}}{2 \Lambda_{1}}\right)^{n}-\left(\frac{\Lambda_{2}+\sqrt{\Lambda_{2}^{2}-4 \Lambda_{1} \Lambda_{3}}}{2 \Lambda_{1}}\right)^{n}+\left(\frac{\Lambda_{3}}{\Lambda_{1}}\right)^{n}\right)}{(-1)^{n+1}\left(k Q_{n}+\left(k-Q_{-n}\right) k^{2}-1\right)}
$$

where

$$
\begin{aligned}
& \Lambda_{1}=k V_{n}-V_{0}, \\
& \Lambda_{2}=k^{\frac{1}{n}}\left(-k V_{n+1}+2 k V_{n}+V_{1}-2 V_{0}\right), \\
& \Lambda_{3}=k^{\frac{2}{n}}\left(k V_{n+2}-2 k V_{n+1}-k V_{n}-V_{2}+2 V_{1}+V_{0}\right) .
\end{aligned}
$$

Proof. By considering identities

$$
\begin{aligned}
\prod_{j=0}^{n-1}\left(x-y \omega^{-j}\right) & =x^{n}-y^{n}, \\
\prod_{j=0}^{n-1}\left(x-y \omega^{-j}+z \omega^{-2 j}\right) & =x^{n}\left(1-\left(\frac{y-\sqrt{y^{2}-4 x z}}{2 x}\right)^{n}-\left(\frac{y+\sqrt{y^{2}-4 x z}}{2 x}\right)^{n}+\left(\frac{z}{x}\right)^{n}\right),
\end{aligned}
$$

and

$$
\left(\left(k^{\frac{1}{n}} \omega^{-j}\right)^{3}+\left(k^{\frac{1}{n}} \omega^{-j}\right)^{2}+2\left(k^{\frac{1}{n}} \omega^{-j}\right)-1\right)=\left(\alpha k^{\frac{1}{n}} \omega^{-j}-1\right)\left(\beta k^{\frac{1}{n}} \omega^{-j}-1\right)\left(\gamma k^{\frac{1}{n}} \omega^{-j}-1\right),
$$

we see that

$$
\prod_{j=0}^{n-1}\left(\left(k^{\frac{1}{n}} \omega^{-j}\right)^{3}+\left(k^{\frac{1}{n}} \omega^{-j}\right)^{2}+2\left(k^{\frac{1}{n}} \omega^{-j}\right)-1\right)=(-1)^{n+1}\left(k Q_{n}+\left(k-Q_{-n}\right) k^{2}-1\right),
$$

and

$$
\prod_{j=0}^{n-1} \Phi_{j}=\Lambda_{1}^{n}\left(1-\left(\frac{\Lambda_{2}-\sqrt{\Lambda_{2}^{2}-4 \Lambda_{1} \Lambda_{3}}}{2 \Lambda_{1}}\right)^{n}-\left(\frac{\Lambda_{2}+\sqrt{\Lambda_{2}^{2}-4 \Lambda_{1} \Lambda_{3}}}{2 \Lambda_{1}}\right)^{n}+\left(\frac{\Lambda_{3}}{\Lambda_{1}}\right)^{n}\right)
$$

where

$$
\begin{aligned}
\omega= & \exp (2 \pi i / n), \\
\Phi_{j}= & k V_{n}-V_{0}-k^{\frac{1}{n}}\left(-k V_{n+1}+2 k V_{n}+V_{1}-2 V_{0}\right) \omega^{-j} \\
& +k^{\frac{2}{n}}\left(k V_{n+2}-2 k V_{n+1}-k V_{n}-V_{2}+2 V_{1}+V_{0}\right) \omega^{-2 j},
\end{aligned}
$$

and

$$
\begin{aligned}
& \Lambda_{1}=k V_{n}-V_{0}, \\
& \Lambda_{2}=k^{\frac{1}{n}}\left(-k V_{n+1}+2 k V_{n}+V_{1}-2 V_{0}\right), \\
& \Lambda_{3}=k^{\frac{2}{n}}\left(k V_{n+2}-2 k V_{n+1}-k V_{n}-V_{2}+2 V_{1}+V_{0}\right) .
\end{aligned}
$$

From Theorem 2.7, we have

$$
\begin{aligned}
\operatorname{det}\left(C_{n}(V)_{k}\right) & =\prod_{j=0}^{n-1} \lambda_{j}\left(C_{n}(V)_{k}\right) \\
& =\prod_{j=0}^{n-1} \frac{\Phi_{j}}{\left(k^{\frac{1}{n}} \omega^{-j}\right)^{3}+\left(k^{\frac{1}{n}} \omega^{-j}\right)^{2}+2\left(k^{\frac{1}{n}} \omega^{-j}\right)-1}
\end{aligned}
$$




$$
\begin{aligned}
& =\frac{\prod_{j=0}^{n-1} \Phi_{j}}{\prod_{j=0}^{n-1}\left(\left(k \frac{1}{n} \omega^{-j}\right)^{3}+\left(k^{\frac{1}{n}} \omega^{-j}\right)^{2}+2\left(k^{\frac{1}{n}} \omega^{-j}\right)-1\right)} \\
& =\frac{\Lambda_{1}^{n}\left(1-\left(\frac{\Lambda_{2}-\sqrt{\Lambda_{2}^{2}-4 \Lambda_{1} \Lambda_{3}}}{2 \Lambda_{1}}\right)^{n}-\left(\frac{\Lambda_{2}+\sqrt{\Lambda_{2}^{2}-4 \Lambda_{1} \Lambda_{3}}}{2 \Lambda_{1}}\right)^{n}+\left(\frac{\Lambda_{3}}{\Lambda_{1}}\right)^{n}\right)}{(-1)^{n+1}\left(k Q_{n}+\left(k-Q_{-n}\right) k^{2}-1\right)},
\end{aligned}
$$

which completes the proof.

We consider two special cases of the above theorem: the determinant of third-order Pell $k$-circulant matrix: $C_{n}(P)_{k}=\operatorname{Circ}_{k}\left(P_{0}, P_{1}, \ldots, P_{n-1}\right)$ and the determinant of third-order PellLucas $k$-circulant matrix: $C_{n}(Q)_{k}=\operatorname{Circ}_{k}\left(Q_{0}, Q_{1}, \ldots, Q_{n-1}\right)$.

Firstly, the following corollary gives the determinant of $C_{n}(P)_{k}$.

Corollary 2.9.1. The determinant of $C_{n}(P)_{k}$ is given by

$$
\operatorname{det}\left(C_{n}(P)_{k}\right)=\frac{\Lambda_{1}^{n}\left(1-\left(\frac{\Lambda_{2}-\sqrt{\Lambda_{2}^{2}-4 \Lambda_{1} \Lambda_{3}}}{2 \Lambda_{1}}\right)^{n}-\left(\frac{\Lambda_{2}+\sqrt{\Lambda_{2}^{2}-4 \Lambda_{1} \Lambda_{3}}}{2 \Lambda_{1}}\right)^{n}+\left(\frac{\Lambda_{3}}{\Lambda_{1}}\right)^{n}\right)}{(-1)^{n+1}\left(k Q_{n}+\left(k-Q_{-n}\right) k^{2}-1\right)}
$$

where

$$
\begin{aligned}
& \Lambda_{1}=k P_{n}, \\
& \Lambda_{2}=k^{\frac{1}{n}}\left(-k P_{n+1}+2 k P_{n}+1\right), \\
& \Lambda_{3}=k^{\frac{2}{n}}\left(k P_{n+2}-2 k P_{n+1}-k P_{n}\right) .
\end{aligned}
$$

Proof. Take $V_{n}=P_{n}, P_{0}=0, P_{1}=1, P_{2}=2$ in Theorem 2.9 .

Secondly, the following corollary gives the determinant of $C_{n}(Q)_{k}$.

Corollary 2.9.2. The determinant of $C_{n}(Q)_{k}$ is given by

$$
\operatorname{det}\left(C_{n}(Q)_{k}\right)=\frac{\Lambda_{1}^{n}\left(1-\left(\frac{\Lambda_{2}-\sqrt{\Lambda_{2}^{2}-4 \Lambda_{1} \Lambda_{3}}}{2 \Lambda_{1}}\right)^{n}-\left(\frac{\Lambda_{2}+\sqrt{\Lambda_{2}^{2}-4 \Lambda_{1} \Lambda_{3}}}{2 \Lambda_{1}}\right)^{n}+\left(\frac{\Lambda_{3}}{\Lambda_{1}}\right)^{n}\right)}{(-1)^{n+1}\left(k Q_{n}+\left(k-Q_{-n}\right) k^{2}-1\right)},
$$

where

$$
\begin{aligned}
& \Lambda_{1}=k Q_{n}-3, \\
& \Lambda_{2}=k^{\frac{1}{n}}\left(-k Q_{n+1}+2 k Q_{n}-4\right), \\
& \Lambda_{3}=k^{\frac{2}{n}}\left(k Q_{n+2}-2 k Q_{n+1}-k Q_{n}+1\right) .
\end{aligned}
$$

Proof. Take $V_{n}=Q_{n}, Q_{0}=3, Q_{1}=2, Q_{2}=6$ in Theorem 2.9. 


\section{Numerical examples}

In this section, we give upper and lower bounds for the spectral norms of $k$-circulant matrix $C_{n}(P)_{k}=\operatorname{Circ}_{k}\left(P_{0}, P_{1}, \ldots, P_{n-1}\right)$ using Theorem 2.8.

\begin{tabular}{|llrrr|}
\hline $\boldsymbol{n}$ & $\boldsymbol{k}$ & Lower bound & $\left\|\boldsymbol{C}_{\boldsymbol{n}}(\boldsymbol{P})_{\boldsymbol{k}}\right\|_{\boldsymbol{2}}$ & \multicolumn{1}{c|}{ Upper bound } \\
\hline 5 & 1 & 14.10673598 & 21.0000000 & 199.49937343 \\
5 & 1.08 & 14.10673598 & 22.191242817 & 152.35274858 \\
5 & 1.7 & 14.10673598 & 32.721630822 & 339.14893503 \\
5 & 2 & 14.10673598 & 38.109512785 & 398.99874687 \\
5 & 4 & 14.10673598 & 74.758299427 & 797.99749373 \\
5 & 5 & 14.10673598 & 93.197313613 & 997.49686716 \\
\hline 8 & 1 & 232.68003782 & 352.000000 & 54140.499998 \\
8 & 1.08 & 232.68003782 & 375.06017982 & 58471.739998 \\
8 & 1.7 & 232.68003782 & 571.06349258 & 92038.850048 \\
8 & 2 & 232.68003782 & 668.84271369 & 108280.99999 \\
8 & 4 & 232.68003782 & 1326.3440329 & 216561.99999 \\
8 & 5 & 232.68003782 & 1655.9173514 & 270702.49999 \\
\hline
\end{tabular}

Table 2. Some upper and lower bounds for the spectral norms of $C_{n}(P)_{k}$ for $n=5,8$ and $|k| \geq 1$

\begin{tabular}{|ccccc|}
\hline $\boldsymbol{n}$ & $\boldsymbol{k}$ & Lower bound & $\left\|\boldsymbol{C}_{\boldsymbol{n}}(\boldsymbol{P})_{\boldsymbol{k}}\right\|_{\boldsymbol{2}}$ & Upper bound \\
\hline 5 & 0.00 & 0.0 & 15.313439017 & 31.543620591 \\
5 & 0.21 & 2.9624145557 & 15.475712850 & 31.543620591 \\
5 & 0.34 & 4.7962902331 & 15.716833208 & 31.543620591 \\
5 & 0.53 & 7.4765700692 & 16.384997574 & 31.543620591 \\
5 & 0.70 & 9.8747151858 & 17.497792306 & 31.543620591 \\
5 & 0.99 & 13.965668620 & 20.856681349 & 31.543620591 \\
\hline 8 & 0.00 & 0.0 & 252.99406315 & 658.11853036 \\
8 & 0.21 & 48.862807942 & 254.92973147 & 658.11853036 \\
8 & 0.34 & 79.111212859 & 258.3008072 & 658.11853036 \\
8 & 0.53 & 123.32042004 & 268.08740786 & 658.11853036 \\
8 & 0.70 & 162.87602647 & 268.08740786 & 658.11853036 \\
8 & 0.99 & 230.35323744 & 349.21360741 & 658.11853036 \\
\hline
\end{tabular}

Table 3. Some upper and lower bounds for the spectral norms of $C_{n}(P)_{k}$ for $n=5,8$ and $|k|<1$.

\section{Acknowledgements}

The author express his sincere thanks to the anonymous referees and the associate editor for their careful reading, suggestions and comments, which improved the presentation of results. 


\section{References}

[1] Alptekin, E. G. (2005). Pell, Pell-Lucas ve Modified Pell sayıları ile tanımlı circulant ve semicirculant matrisler. [PhD thesis, Selçuk Üniversitesi Fen Bilimleri Enstitüsü]. Selcuk University Digital Archive. Retrieved from: http://hdl.handle.net/ $123456789 / 5959$

[2] Cline, R. E., Plemmons, R. J., \& Worm, G. (1974). Generalized Inverses of Certain Toeplitz Matrices. Linear Algebra and its Applications 8, 25-33.

[3] Coskun, A., \& Taskara, N. (2018). On the Some Properties of Circulant Matrices with Third Order Linear Recurrent Sequences. Mathematical Sciences and Applications E-Notes, 6(1), $12-18$.

[4] Davis, P. J. (1979). Circulant Matrices, John Wiley \& Sons, New York.

[5] Deveci, O., Karaduman, E., \& Campbell, C. M. (2007). The Fibonacci-Circulant Sequences and Their Applications. Iranian Journal of Science and Technology Transaction A-Science, 41(A4), 1033-1038.

[6] Deveci, O. (2018). On The Fibonacci-Circulant p-Sequences. Utilitas Mathematica, 108, $107-124$.

[7] Horn, R. A., \& Johnson, C. R. (2012). Matrix Analysis, Cambridge University Press, Cambridge, 2nd edition.

[8] Horn, R. A., \& Johnson, C. R. (1991). Topics in Matrix Analysis, Cambridge University Press, Cambridge.

[9] Kızılateş, C., \& Tuglu, N. (2018). On the Norms of Geometric and Symmetric Geometric Circulant Matrices with the Tribonacci Number. Gazi University Journal of Science, 31(2), $555-567$.

[10] Kizılateş, C., \& Tuglu, N. (2016). On the Bounds for the Spectral Norms of Geometric Circulant Matrices. Journal of Inequalities and Applications, 2016(1), Article ID 312.

[11] Özkoç, A., \& Ardiyok, E. (2016). Circulant and Negacyclic Matrices Via Tetranacci Numbers. Honam Mathematical Journal, 38(4), 725-738.

[12] Pacheenburawana, A., \& Sintunavarat, W. (2018). On the Spectral Norms of $r$-Circulant Matrices with the Padovan and Perrin Sequences. Journal of Mathematical Analysis, 9(3), $110-122$.

[13] Pethe, S. (1988). Some Identities for Tribonacci sequences. The Fibonacci Quarterly, 26(2), 144-151.

[14] Polatl1, E. (2019). On the Bounds for the Spectral Norms of $r$-Circulant Matrices with a Type of Catalan Triangle Numbers. Journal of Science and Arts, 3(48), 575-578. 
[15] Radičić, B. (2019). On k-Circulant Matrices Involving the Jacobsthal Numbers. Revista De La Union Matematica Argentina, 60(2), 431-442.

[16] Raza, Z., Riaz, M., \& Ali, M. A. (2015). Some Inequalities on the Norms of Special Matrices with Generalized Tribonacci and Generalized Pell-Padovan Sequences. arXiv, http://arxiv.org/abs/1407.1369v2.

[17] Raza, Z., \& Ali, M. A. (2014). On the Norms of Circulant, $r$-Circulant, Semi-Circulant and Hankel Matrices with Tribonacci Sequence. arXiv, http://arxiv.org/abs/1407.1369v1.

[18] Sintunavarat, W. (2016). The Upper Bound Estimation for the Spectral Norm of $r$-Circulant and Symmetric $r$-Circulant Matrices with the Padovan Sequence, Journal of Nonlinear Sciences and Applications, 9, 92-101.

[19] Sloane, N. J. A. The on-line encyclopedia of integer sequences. Available online at: http: //oeis.org/.

[20] Soykan Y. (2020). Generalized Tribonacci Numbers: Summing Formulas. International Journal of Advances in Applied Mathematics and Mechanics, 7(3), 57-76.

[21] Soykan Y. (2020). A Study On Generalized $(r, s, t)$-Numbers. MathLAB Journal, 7, 101-129.

[22] Soykan, Y. (2019). On Generalized Third-Order Pell Numbers. Asian Journal of Advanced Research and Reports, 6(1), 1-18.

[23] Soykan, Y. (2020). A Closed Formula for the Sums of Squares of Generalized Tribonacci numbers. Journal of Progressive Research in Mathematics, 16(2), 2932-2941.

[24] Soykan, Y. (2020). On the Sums of Squares of Generalized Tribonacci Numbers: Closed Formulas of $\sum_{k=0}^{n} x^{k} W_{k}^{2}$. Archives of Current Research International, 20(4), 22-47.

[25] Soykan, Y. (2020). Summing Formulas For Generalized Tribonacci Numbers. Universal Journal of Mathematics and Applications, 3(1), 1-11.

[26] Soykan, Y. (2021). A Study On the Sums of Squares of Generalized Tribonacci Numbers: Closed Form Formulas of $\sum_{k=0}^{n} k x^{k} W_{k}^{2}$. Journal of Scientific Perspectives, 5(1), 1-23.

[27] Soykan, Y. (2020). A Study on Sum Formulas for Generalized Tribonacci Numbers: Closed Forms of the Sum Formulas $\sum_{k=0}^{n} k x^{k} W_{k}$ and $\sum_{k=1}^{n} k x^{k} W_{-k}$. Journal of Progressive Research in Mathematics, 17(1), 1-40.

[28] Soykan, Y. (2020). Formulae For The Sums of Squares of Generalized Tribonacci Numbers: Closed Form Formulas of $\sum_{k=0}^{n} k W_{k}^{2}$. IOSR Journal of Mathematics, 16(4), 1-18.

[29] Soykan, Y. (2020). On Sum Formulas for Generalized Tribonacci Sequence. Journal of Scientific Research \& Reports, 26(7), 27-52. 
[30] Tuglu, N., \& Kızılateş, C. (2015). On the Norms of Circulant and $r$-Circulant Matrices with the Hyperharmonic Fibonacci Numbers. Journal of Inequalities and Applications, 2015, Article ID 253.

[31] Turkmen, R., \& Gökbaş, H. (2016). On the Spectral Norm of $r$-Circulant Matrices with the Pell and Pell-Lucas Numbers. Journal of Inequalities and Applications, 2016, Article ID 65.

[32] Uygun, Ş. (2016). Some Bounds for the Norms of Circulant Matrices with the $k$-Jacobsthal and $k$-Jacobsthal Lucas Numbers. Journal of Mathematics Research, 8(6), 133-138.

[33] Uygun, Ş., \& Yaşamalı, S. (2017). On the Bounds for the Norms of Circulant Matrices with the Jacobsthal and Jacobsthal-Lucas Numbers. Notes on Number Theory and Discrete Mathematics, 23(1), 91-98.

[34] Uygun, Ş., \& Yaşamal1, S. (2017). On the Bounds for the Norms of $r$-Circulant Matrices with the Jacobsthal and Jacobsthal-Lucas Numbers. International Journal of Pure and Applied Mathematics, 112(1), 93-102.

[35] Zielke, G. (1988). Some Remarks on Matrix Norms, Condition Numbers, and Error Estimates for Linear Equations. Linear Algebra and its Applications, 110, 29-41. 